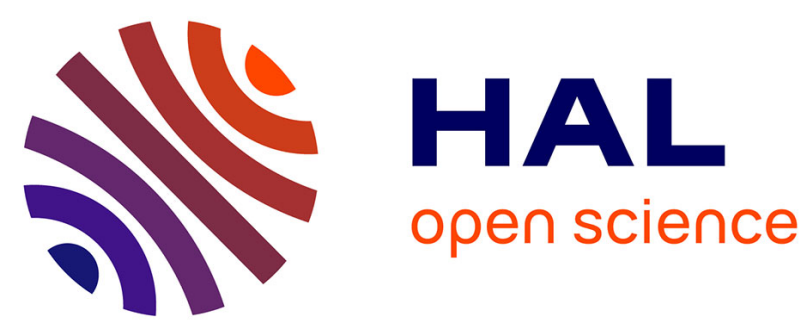

\title{
Differential Importance Measure of Markov Models Using Perturbation Analysis
}

Phuc Do Van, Anne Barros, Christophe Bérenguer

\section{To cite this version:}

Phuc Do Van, Anne Barros, Christophe Bérenguer. Differential Importance Measure of Markov Models Using Perturbation Analysis. European Safety and Reliability Conference (ESREL 2009), Sep 2009, Prague, Czech Republic. pp.981-987. hal-00705213

\section{HAL Id: hal-00705213 https://hal.science/hal-00705213}

Submitted on 7 Jun 2012

HAL is a multi-disciplinary open access archive for the deposit and dissemination of scientific research documents, whether they are published or not. The documents may come from teaching and research institutions in France or abroad, or from public or private research centers.
L'archive ouverte pluridisciplinaire HAL, est destinée au dépôt et à la diffusion de documents scientifiques de niveau recherche, publiés ou non, émanant des établissements d'enseignement et de recherche français ou étrangers, des laboratoires publics ou privés. 


\title{
Differential Importance Measure of Markov Models Using Perturbation Analysis
}

\author{
P. Do Van, A. Barros \& C. Bérenguer \\ Université de technologie de Troyes/ICD FRE CNRS 2848, Troyes, France
}

\begin{abstract}
Reliability importance measures providing information about the importance of components on the system performance (reliability, maintainability, safety, or any performance metrics of interest) have been widely used in reliability studies and risk analysis. In this paper, the differential importance measure (DIM) introduced recently for use in risk-informed decision-making is extended to Markov reliability models. This allows to qualify the relative contribution of a component (or a group of components), as well as a state (or a group of states) on the total variation of system performance. The estimation of DIM at steady state from the operating feedback data by using perturbation analysis is also investigated. A numerical example of a dynamic system is finally introduced to illustrate the use of DIM, as well as the advantages of proposed approach.
\end{abstract}

\section{INTRODUCTION}

Reliability importance measures providing information about the importance of components on the system performance (reliability, maintainability, safety, or any performance metrics of interest) have been widely used in reliability studies and risk analysis. They are useful tools to identify design weaknesses or operation bottlenecks and to suggest optimal modifications for system upgrade. Recently, a new importance measure, called Differential Importance Measure (DIM), has been introduced for use in riskinformed decision-making (Borgonovo and Apostolakis 2001; Borgonovo et al. 2003; Vinod et al. 2003; Marseguerra and Zio 2004). The DIM is defined as a first-order sensitivity measure that ranks the parameters of the risk model according to the fraction of the total change in the risk metric that is due to a small change in the parameters' values, taken one at a time. However, this importance measure is often only applicable on a limited class of system with independent components.

The first objective of this paper is to develop the differential importance measure in the context of dynamic systems including inter-component, functional dependencies, or more generally, systems described by Markov models. In such systems, the (un)availability of a component does not depend only on its characteristics but also on other system parameters, and its (un)availability in the system can be different from its (un)availability out of the system, see (Ou and Bechta-Dugan 2003). In this context, the partial derivatives with respect to the system parameters, or more generally, the directional deriva- tives (Do Van et al. 2008c), rather than to the components'(un)availability, appears to be more relevant and is often preferred for design purposes. Hence, we develop the DIM based on the directional derivative in the direction defined by a matrix in an appropriate space. This allows to qualify the relative contribution of a component (or a group of components), as well as a state (or a group of states) on the total variation of system performance.

In the framework of Markov models, one has shown in (Do Van et al. 2008c) that perturbation analysis and one of its extension presented in (Cao and Chen 1997) can be very well adapted to reliability or maintenance problems at steady state. The second objective of the present paper is to show how the perturbation technique can be used in order to estimate the DIM of Markov models at steady state by using only a single sample path. Moreover, when perturbation parameters have been estimated, the DIM of any direction can be easily obtained without additional calculations. As a consequence, different maintenance policies or inspection schedules can be easily tested and optimized.

This paper is organized as follows: Section 2 is devoted to the development of DIM in the context of Markov models. The analytical calculation of DIM based on the directional derivative is also investigated. Section 3 focuses on the estimation of DIM at steady state from a single sample path of Markov process using the perturbation analysis technique. A simple numerical example is introduced in Section 4 to illustrate the use of DIM in reliability studies, as well as the advantages of proposed approaches. Finally, Sec- 
tion 5 presents the conclusions drawn from this work.

\section{NOTATION LIST}

$\lambda_{i}, \mu_{i} \quad$ failure and repair rate of component $i$

M transition rate matrix of Markov models

$\mathbf{M}^{\sharp} \quad$ group inverse of $\mathbf{M}$

$X^{l} \quad$ irreducible homogenous Markov process with the initial state $l$, and the transition rate matrix $\mathbf{M}$

Q directional perturbation matrix

$\boldsymbol{\pi}$ row vector of steady-state probabilities

$A \quad$ asymptotic performance measure of the system

$\delta^{I} A \quad$ first-order variation of system performance measure $A$

DIM differential importance measure

$\frac{d A}{d \mathbf{Q}_{i}} \quad$ directional derivative of $A$ in the direction $\mathbf{Q}_{i}$

$\delta_{i} \quad$ amount of variation in the direction $\mathbf{Q}_{i}$

$d_{i j} \quad$ perturbation realization factor

D realization matrix

\section{DIFFERENTIAL IMPORTANCE MEASURE}

Markov processes have been widely used to analyse and assess the performances (reliability, availability, maintainability, production capacity, etc...) of many complex dynamical systems with inter-component and functional dependencies (cold spare, shared load, shared resources, ...). This section explores the development of the differential importance measure in the context of Markov models at steady state.

Consider a n-components dynamic system described by an irreducible homogenous Markov process $X^{l}=\left\{X_{t}^{l}, t \geq 0\right\}$ (initial state $l$ ) with finite state space $E$ and the transition rate matrix M. This Markov process is ergodic and a single stationary distribution exists (Ross 1996). Let a row vector $\boldsymbol{\pi}=\left(\pi_{1}, \pi_{2}, \ldots\right)$ be the vector of steady state probabilities (stationary distribution vector). ChapmanKolmogorov equations at steady state can be written as:

$$
\pi \mathbf{M}=0
$$

Let $f$ be the performance function associated with the system states. The system performance (availability, production capacity, etc.) is usually considered as the expected performance function.

$$
A=\mathbb{E}_{\boldsymbol{\pi}}(f)=\sum_{i \in E} \pi_{i} f(i)=\boldsymbol{\pi} \boldsymbol{f}
$$

where $\mathbb{E}_{\boldsymbol{\pi}}$ denotes the expected with respect to the steady state probabilities $\boldsymbol{\pi}$, and $\boldsymbol{f}=\left(f_{1}, f_{2}, \ldots,\right)^{T}$ a column vector with $f_{i}=f(i)$. For example, if one considers the system availability then $f_{i}=1$ when system is operational in state $\mathrm{i}$ and $f_{i}=0$ otherwise.

\subsection{Definition of DIM}

Consider now a perturbation on the transition rate matrix $\mathbf{M}$ of the Markov process. Consequently, the perturbed transition rates matrix is :

$$
\mathbf{M}_{\delta}=\mathbf{M}+\delta \mathbf{Q},
$$

where $\delta$ is a real number and $\mathbf{Q}$ is a matrix representing the direction of perturbation. If an entry $M_{i j}=0$, the corresponding entry $Q_{i j}$ must then equal 0 . An entry $Q_{i j}=\alpha$ different from 0 indicates that the transition rate from state $i$ to state $j$ is perturbed by an amount $\alpha \delta$. The only condition on the structure of $\mathbf{Q}$ to ensure that the matrix $\mathbf{M}_{\delta}$ remains a transition rate matrix of a Markov process is that the sum of each row of $\mathbf{Q}$ equals 0 .

The matrix $\mathbf{Q}$ can be used to describe the change of one parameter, a group of parameters, more generally, the change in any direction of transition rates.

Example: Consider a system consisting of two independent units $\mathrm{C} 1$ and $\mathrm{C} 2$ in a parallel structure with constant failure rates $\lambda_{1}, \lambda_{2}$ and constant repair rates $\mu_{1}, \mu_{2}$ (see Figure 1).

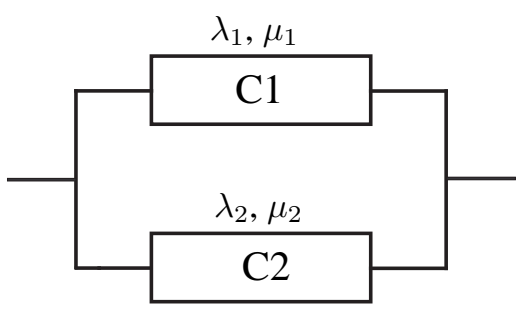

Figure 1. A parallel structure of 2 units

The transition rate matrix of this system is given by:

$\mathbf{M}=\left(\begin{array}{cccc}-\lambda_{1}-\lambda_{2} & \lambda_{1} & \lambda_{2} & 0 \\ \mu_{1} & -\mu_{1}-\lambda_{2} & 0 & \lambda_{2} \\ \mu_{2} & 0 & -\mu_{2}-\lambda_{1} & \lambda_{1} \\ 0 & \mu_{2} & \mu_{1} & -\mu_{1}-\mu_{2}\end{array}\right)$

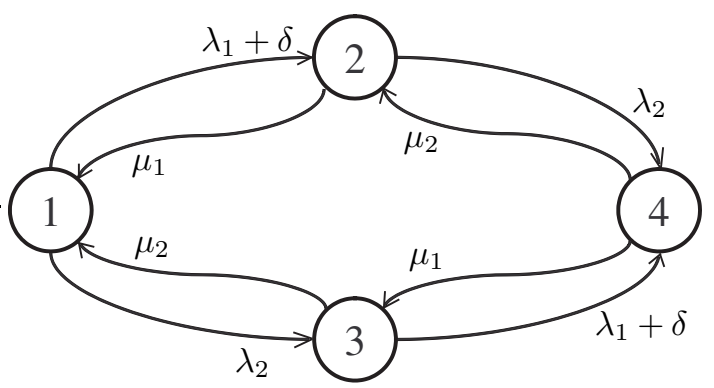

State $1: \mathrm{C} 1 \mathrm{C} 2 \quad$ State $2: \overline{\mathrm{C} 1 \mathrm{C} 2}$

State $3: \mathrm{C} 1 \overline{\mathrm{C} 2} \quad$ State $4: \overline{\mathrm{C} 1} \overline{\mathrm{C} 2}$

Figure 2. Markov diagram with perturbation on $\lambda_{1}$

The state diagram of this system is sketched in Figures 2 and 3 for two different types of perturbations. 
Figure 2 sketches the Markov graph with a perturbation on one specific parameter, namely $\lambda_{1}$, which corresponds to the directional perturbation matrix $\mathbf{Q}_{\lambda_{1}}$.

$$
\mathbf{Q}_{\lambda_{1}}=\left(\begin{array}{cccc}
-1 & 1 & 0 & 0 \\
0 & 0 & 0 & 0 \\
0 & 0 & -1 & 1 \\
0 & 0 & 0 & 0
\end{array}\right) ; \mathbf{Q}_{3}=\left(\begin{array}{cccc}
0 & 0 & 0 & 0 \\
0 & 0 & 0 & 0 \\
3 & 0 & -1 & -2 \\
0 & 0 & 0 & 0
\end{array}\right)
$$

Figure 3 presents the state diagram modified by a perturbation on the exit transition rates of one specific state, namely state number 3 . This perturbation corresponds to the directional perturbation matrix $\mathbf{Q}_{3}$.

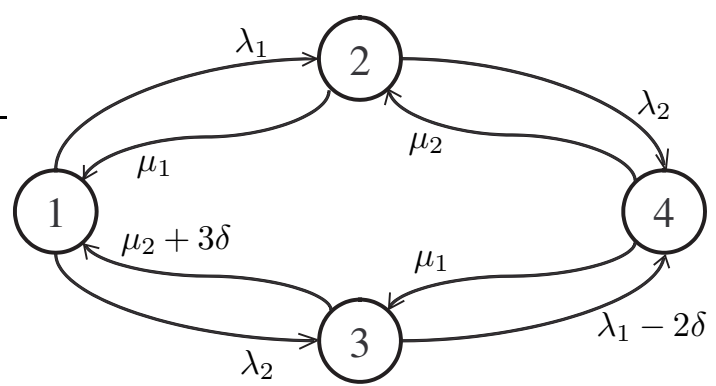

Figure 3. Perturbation on the exit transition rates of state 3

If one considers the case in which the transition rate matrix is perturbed in $K$ different directions $\left(\mathbf{Q}_{1}, \mathbf{Q}_{2}, \cdots, \mathbf{Q}_{K}\right.$ with $\mathbf{Q}_{i, i=1, \ldots K}$ could be relative to a parameter, a state, etc), the perturbed transition rates matrix is then:

$$
\begin{aligned}
\mathbf{M}_{\delta} & =\mathbf{M}+\delta_{1} \mathbf{Q}_{1}+\delta_{2} \mathbf{Q}_{2}+\ldots+\delta_{K} \mathbf{Q}_{K} \\
& =\mathbf{M}+\sum_{i=1}^{K} \delta_{i} \mathbf{Q}_{i},
\end{aligned}
$$

with $\delta_{i}(i=1, \ldots, K)$ is an amount of variation in the direction $\mathbf{Q}_{i}$.

\subsection{Definition of DIM}

The variations in transition rate matrix described in Equation (4) may lead to a variation of the system performance, noted $\delta A$. If the changes of parameters are small enough, the variation of the system performance $\delta A$ can be approximated by the first-order contribution $\delta^{I} A$, see (Borgonovo and Apostolakis 2001):

$$
\delta A \simeq \delta^{I} A=\sum_{i=1}^{K} \delta_{i} \frac{d A}{d \mathbf{Q}_{i}}=\sum_{i=1}^{K} \delta^{I} A_{i},
$$

where:

- $\frac{d A}{d \mathbf{Q}_{i}}$ is the directional derivative of $A$ in the direction $\mathbf{Q}_{i}$ (Cao and Chen 1997). It is used as an importance measure, namely multi directional importance measure, in reliability sensitivity analysis (Do Van et al. 2008c; Do Van et al. 2008a),
- $\delta^{I} A_{i}=\delta_{i} \frac{d A}{d \mathbf{Q}_{i}}$ is the first-order contribution of the change in transition rates matrix with respect to the direction $\mathbf{Q}_{i}$ and the amount $\delta_{i}$.

In order to qualify the relative contribution due to the change in a direction on the total variation of system performance, the differential importance measure (DIM (Borgonovo and Apostolakis 2001; Borgonovo et al. 2003)) based on the partial derivatives. More precisely, the DIM in the direction $\mathbf{Q}_{i}$ can be defined as:

$$
\operatorname{DIM}\left(\mathbf{Q}_{i}\right)=\frac{\delta^{I} A_{i}}{\delta^{I} A}=\frac{\delta_{i} \frac{d A}{d \mathbf{Q}_{i}}}{\sum_{j=1}^{K} \delta_{j} \frac{d A}{d \mathbf{Q}_{j}}} .
$$

If the direction $\mathbf{Q}_{i}$ relates to a component (or a group of components), a state (or a group of states), the DIM in the direction $\mathbf{Q}_{i}$ represents then the relative contribution, on the total variation of the system performance, of a component (or a group of components), a state (or a group of states) respectively. An application example of DIM in reliability sensitivity analysis can be found in Section 4.

By definition, the DIM shares two interesting properties:

Property 1: Additivity. If we are interested in the DIM in the subset of directions $\mathbf{Q}_{i}, \mathbf{Q}_{j}, \ldots \mathbf{Q}_{s}$. Then:

$$
\begin{gathered}
\operatorname{DIM}\left(\mathbf{Q}_{i}, \mathbf{Q}_{j}, \ldots, \mathbf{Q}_{s}\right)=\frac{\delta^{I} A_{i, j, . . s}}{\delta^{I} A} \\
=\frac{\delta_{i} \frac{d A}{d \mathbf{Q}_{i}}+\delta_{j} \frac{d A}{d \mathbf{Q}_{j}}+\ldots+\delta_{s} \frac{d A}{d \mathbf{Q}_{s}}}{\sum_{j=1}^{K} \delta_{j} \frac{d A}{d \mathbf{Q}_{j}}}
\end{gathered}
$$$$
=\operatorname{DIM}^{1}\left(\mathbf{Q}_{i}\right)+\operatorname{DIM}^{1}\left(\mathbf{Q}_{j}\right)+\ldots+\operatorname{DIM}^{1}\left(\mathbf{Q}_{s}\right) .
$$

This relationship shows that the DIM is additive. This important property can be used to the DIM relative of a group components given the DIM relative of each component in this group.

Property 2: The sum of the DIMs of all directions equals unity, that is:

$$
\operatorname{DIM}\left(\mathbf{Q}_{1}\right)+\operatorname{DIM}^{1}\left(\mathbf{Q}_{2}\right)+\ldots+\operatorname{DIM}^{1}\left(\mathbf{Q}_{K}\right)=1 .
$$

This property can be used to determine the DIM of a direction from the other.

\subsection{Evaluation of DIM}

Theorem 1 If the transition rate matrix is perturbed in the directional matrix $\mathbf{Q}$, i.e. Equation (3) holds, 
the directional derivative of $A$ in this direction $\mathbf{Q}$ can be then written as the following:

$$
\frac{d A}{d \mathbf{Q}}=-\boldsymbol{\pi} \mathbf{Q} \mathbf{M}^{\sharp} \boldsymbol{f},
$$

where $\mathbf{M}^{\sharp}$ is the generalized inverse (or group inverse) of $\mathbf{M}$ defined as: $\mathbf{M}^{\sharp}=(\mathbf{M}+e \boldsymbol{\pi})^{-1}-e \boldsymbol{\pi}$, with $\boldsymbol{e}=(1,1, \ldots, 1)^{\top}$.

Proof: the demonstration of this theorem can be found in (Cao and Chen 1997; Do Van et al. 2008c).

By using this result, Equation (6) can be expressed as:

$$
\operatorname{DIM}\left(\mathbf{Q}_{i}\right)=\frac{-\delta_{i} \boldsymbol{\pi} \mathbf{Q}_{i} \mathbf{M}^{\sharp} \boldsymbol{f}}{-\sum_{j=1}^{K} \delta_{j} \boldsymbol{\pi} \mathbf{Q}_{j} \mathbf{M}^{\sharp} \boldsymbol{f}}=\frac{\boldsymbol{\pi} \mathbf{Q}_{i}^{\delta} \mathbf{M}^{\sharp} \boldsymbol{f}}{\boldsymbol{\pi} \mathbf{Q}_{\sum}^{\delta} \mathbf{M}^{\sharp} \boldsymbol{f}},
$$

where:

- $\mathbf{Q}_{i}^{\delta}=\delta_{i} \mathbf{Q}_{i}$,

- $\mathbf{Q}_{\Sigma}^{\delta}=\sum_{i=1}^{K} \mathbf{Q}_{i}^{\delta}$.

This formula shows that the DIM of different directions can be easily obtained by changing only the directional matrix without additional calculations. Moreover, the DIM of a group of directions can be directly calculated as:

$$
\operatorname{DIM}\left(\mathbf{Q}_{i}, \mathbf{Q}_{j}, \ldots, \mathbf{Q}_{s}\right)=\frac{\boldsymbol{\pi} \mathbf{Q}_{i, j, \ldots s}^{\delta} \mathbf{M}^{\sharp} \boldsymbol{f}}{\boldsymbol{\pi} \mathbf{Q}_{\Sigma}^{\delta} \mathbf{M}^{\sharp} \boldsymbol{f}},
$$

with $\mathbf{Q}_{i, j, \ldots s}^{\delta}=\mathbf{Q}_{i}^{\delta}+\mathbf{Q}_{j}^{\delta}+\ldots+\mathbf{Q}_{s}^{\delta}$.

Note well that the DIM can be easily evaluated by using Equation (6), it does not however account for the higher-order effects of simultaneous changes of several parameters, and it can therefore be used only when the changes of parameters are small enough. The idea of second-order extension of the DIM, is considered in (Zio and Podofillini 2006), and the higher-order extension can be found in (Do Van et al. 2008b).

\section{ESTIMATION OF DIM USING PERTURBA- TION ANALYSIS}

From a practical point of view, the analytical method developed in the previous section can be used to evaluate DIM. This method may however lead to some difficulties. The reliability behaviour (failure rate, repair rate) of some components of the system may be unknown, and the transition rate matrix of Markov process modeling the system may evenly be unknown. In these cases, the analytical method may be unusable. Moreover, this method could be computationally burdensome or highly inefficient when the state space dimension is too high. It is therefore interesting to have an effective method for estimating DIM from the observed data of the system, i.e. from a nominal trajectory of the corresponding Markov process. (Do Van, Barros, and Berenguer 2008c) shows that perturbation analysis (AP) can be a promising approach since the AP allows to estimate the directional derivative from a single sample path of Markov process.

\subsection{Directional derivative estimate}

Assume that the transition rate matrix $\mathbf{M}$ of Markov process is unknown and that only an observed sample path of this Markov process is available. Cao showed in (Cao and Chen 1997) by using perturbation analysis that the directional derivative of the steady state performance measure $A$ with respect to the direction $\mathbf{Q}$ can be written as:

$$
\frac{d A}{d \mathbf{Q}}=-\boldsymbol{\pi} \mathbf{Q D} \boldsymbol{\pi}^{T}
$$

where $\mathbf{D}=\left[d_{i j}\right]_{i, j \in E}$ is a realization matrix. $d_{i j}$, called a perturbation realization factor, can be estimated by:

$$
d_{i j}=\mathbb{E}\left\{\int_{0}^{\Gamma^{j}(i)} f\left(X_{t}^{l}\right) d t\right\}-A * \mathbb{E}\left\{\Gamma^{j}(i)\right\},
$$

with:

- $\mathbb{E}$ is the mathematical expectation,

- $\Gamma^{j}(i)=\inf \left\{t, t>0 / X_{t}^{j}=i\right\}$ is the first passage time from state $j$ to state $i$ of Markov process $X_{t}^{l}$.

The first passage time $\Gamma^{j}(i)$ and the stationary probabilities vector $\boldsymbol{\pi}$ (and consequently $A$ ) can be estimated from $X^{j}=\left(X_{t}^{j}, t \geq 0\right)$ as follows. A Markov process $X_{t}^{l}$ (initial state $l$ and transition matrix $\mathbf{M})$ is observed with the two time sequences $\left\{j_{s}\right\}$ and $\left\{i_{s}\right\}$ so that:

- $i_{0}=0$,

- $j_{s}=$ is the time upon which $X_{t}^{l}$ is in state $j$ for the first time after time $i_{s-1}, s \geq 1$,

- $i_{s}=$ is the time upon which $X_{t}^{l}$ is in state $i$ for the first time after time $j_{s}, s \geq 1$.

$\left\{j_{s}\right\}$ and $\left\{i_{s}\right\}$ are well defined on a sample path. Now define:

- $L_{s}^{j}(i)=i_{s}-j_{s}$ for $s \geq 1$ (see Figure 4$)$,

- $R_{s}=\int_{j_{s}}^{i_{s}-1} f\left(X_{t}^{l}\right) d t$

- $\epsilon^{k}(v)$ such that $\epsilon^{k}(v)=1$ if $v=k$ and $\epsilon^{k}(v)=0$ otherwise. 


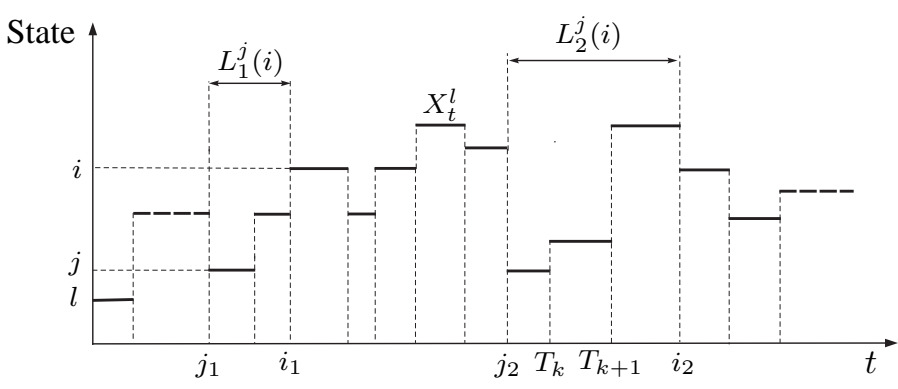

Figure 4. Illustration of $L_{s}^{j}(i)$ on observed single sample path $X^{l}$.

Then $\Gamma^{j}(i), \int_{0}^{\Gamma^{j}(i)}\left[f\left(X_{t}^{j}\right) d t\right]$, and $A$ are estimated by $\hat{\Gamma}^{j}(i), \hat{I}$, and $\hat{A}$ respectively :

$$
\begin{aligned}
& \hat{\Gamma}^{j}(i)=\frac{1}{n} \sum_{s=1}^{n} L_{s}^{j}(i), \\
& \hat{I}=\frac{1}{n} \sum_{s=1}^{n} R_{s}, \\
& \hat{\pi}_{i}=\frac{1}{T_{N}} \int_{0}^{T_{N}} \epsilon^{i}\left(X_{t}^{l}\right) d t, \text { for all } i \in E, \\
& \hat{A}=\hat{\boldsymbol{\pi}} \boldsymbol{f}, \text { with } \hat{\boldsymbol{\pi}}=\left(\hat{\pi}_{1}, \hat{\pi}_{2}, \ldots\right),
\end{aligned}
$$

where $n$ is the number of observed interval $\left[j_{s}, i_{s}\right], N$ is the total number of observed transitions and $T_{k}$ is the $k$-th transition time. Consequently:

$$
\hat{d}_{i j}=\hat{I}-\hat{A} * \hat{\Gamma}^{j}(i) .
$$

The convergence of these estimators based on the realization matrix has been studied and proved by $\mathrm{CaO}$ in (Cao and Chen 1997).

The estimation of the matrix $\mathbf{D}=\left[d_{i j}\right]_{i, j \in E}$ can also be computationally burdensome because it must be made for each couple $(i, j)$ (complexity of order $O\left(m^{2}\right)$ ). That is why an approximate estimate (with potential vector) is proposed in (Cao and Wan 1998) which reduces the complexity of the calculation to the order $O(m)$.

Hence, thanks to the ergodicity of the process under consideration, the perturbation realization leads to an estimate of the directional derivative that:

- can be evaluated from a single sample path. This is very interesting from a practical point of view for on-line performance optimization, when the parameters are impossible to change intentionally, or when the simulation of each perturbed path is computationally burdensome;

- can be evaluated without knowing the infinitesimal generator $\mathbf{M}$ of the Markov process;

- can be evaluated in any direction by changing only the directional matrix $\mathbf{Q}$.

\subsection{DIM estimate}

By using this result showed in Equation (12), Equation (6) can be written as the following:

$$
\operatorname{DIM}\left(\mathbf{Q}_{i}\right)=\frac{-\delta_{i} \boldsymbol{\pi} \mathbf{Q}_{i} \mathbf{D} \boldsymbol{\pi}^{T}}{-\sum_{j=1}^{K} \delta_{j} \boldsymbol{\pi} \mathbf{Q}_{j} \mathbf{D} \boldsymbol{\pi}^{T}}=\frac{\boldsymbol{\pi} \mathbf{Q}_{i}^{\delta} \mathbf{D} \boldsymbol{\pi}^{T}}{\boldsymbol{\pi} \mathbf{Q}_{\sum}^{\delta} \mathbf{D} \boldsymbol{\pi}^{T}}
$$

The estimation of DIM is then:

$$
\operatorname{DIM}\left(\mathbf{Q}_{i}\right)=\frac{\hat{\boldsymbol{\pi}} \mathbf{Q}_{i}^{\delta} \hat{\mathbf{D}} \hat{\boldsymbol{\pi}}^{T}}{\hat{\boldsymbol{\pi}} \mathbf{Q}_{\Sigma}^{\delta} \hat{\mathbf{D}} \hat{\boldsymbol{\pi}}^{T}} .
$$

$\hat{\boldsymbol{\pi}}=\left(\hat{\pi}_{1}, \hat{\pi}_{2}, \ldots\right)^{T}, \hat{\mathbf{D}}=\left[\hat{d}_{i j}\right]_{i, j \in E}$ can be estimated from a single sample path by using Equations (16) and (18) respectively. It is clear that the estimation of DIM can be obtained, by using perturbation analysis technique, from an observed trajectory of system without knowing the transition rate matrix of Markov process.

\section{NUMERICAL EXAMPLE}

The purpose of this section is to show how the differential importance measure DIM can be used in reliability sensitivity analysis of Markov models through a simple example. Performance measure considered in the study is the system availability at steady state. The use of perturbation analysis is also considered in order to estimate the DIM's measures from a single sample path of Markov process.

Figure 5 represents the structure of a simple system with 3 units $(\mathrm{C} 1, \mathrm{C} 2, \mathrm{C} 3)$. Unit $\mathrm{C} 3$ is in cold redundancy with $\mathrm{C} 2$. $\mathrm{C} 2$ is thus the main operating unit of group $(\mathrm{C} 2, \mathrm{C} 3)$ : as soon as $\mathrm{C} 2$ is repaired, $\mathrm{C} 3$ stops and priority is always given to the repair of $\mathrm{C} 2$, see Figure 6.

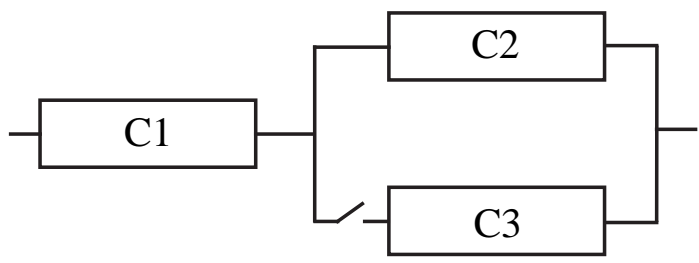

Figure 5. System structure.

The operational mode of the system is described in Table 2 where "O" denotes an operating state, "S" denotes a standby state, and " $F$ " denotes a failed state. The corresponding Markov process is sketched in Figure 6. Table 1 gives the values of failure rates $\lambda_{i}$, the repair rates $\mu_{i}(i=1,2,3)$.

According to the Markov diagram shown in Figure 6 , the system availability at steady state is :

$$
A=\pi_{1}+\pi_{3}+\pi_{4}=\boldsymbol{\pi} \boldsymbol{f}, \text { with } \boldsymbol{f}=(1,0,1,1,0,0,0)^{T} .
$$

By resolving the Chapman-Kolmogorov equations at steady state (1), one obtains finally the steady state availability $A=0,7895$. 
Table 1. Failure and repair rates.

\begin{tabular}{ccc}
\hline Unit & $\lambda_{i}$ & $\mu_{i}$ \\
\hline $\mathrm{C} 1$ & $1 \mathrm{e}-3$ & $1 \mathrm{e}-2$ \\
$\mathrm{C} 2$ & $5 \mathrm{e}-3$ & $1 \mathrm{e}-2$ \\
$\mathrm{C} 3$ & $5 \mathrm{e}-3$ & $1 \mathrm{e}-2$ \\
\hline
\end{tabular}

Table 2. System states

\begin{tabular}{ccccc}
\hline & \multicolumn{3}{c}{ Component } & \\
\cline { 2 - 3 } State & C1 & C2 & C3 & System \\
\hline 1 & O & O & S & O \\
2 & F & O & S & F \\
3 & O & F & O & O \\
4 & O & O & F & O \\
5 & F & F & O & F \\
6 & O & F & F & F \\
7 & F & O & F & F \\
\hline
\end{tabular}

Assume now that all component's failure rates are simultaneously changed. For example, all of components in an aircraft subsystem would presumably be subject to many of the same stress vibration from the engines, shock of landing, irregularities in the power supplied, etc. To illustrate the application of DIM, two following scenarios of changes could be proposed:

- uniform changes (H1): $\delta_{\lambda_{1}}=\delta_{\lambda_{2}}=\delta_{\lambda_{3}}=\delta$. If one substitutes this relationship into Equation (6), the DIM's measures do not then depend on the magnitude of change $\delta$, provided that we consider small changes.

- proportional relative changes $(\mathrm{H} 2)$ :

$$
\frac{\delta_{\lambda_{1}}}{\lambda_{1}}=\frac{\delta_{\lambda_{2}}}{\lambda_{2}}=\frac{\delta_{\lambda_{3}}}{\lambda_{3}}=\omega
$$

Similarly, by substituting this relationships into Equation (6), the DIM's measures do not depend on the percentage of change $\omega$ under the hypothesis that the changes are small.

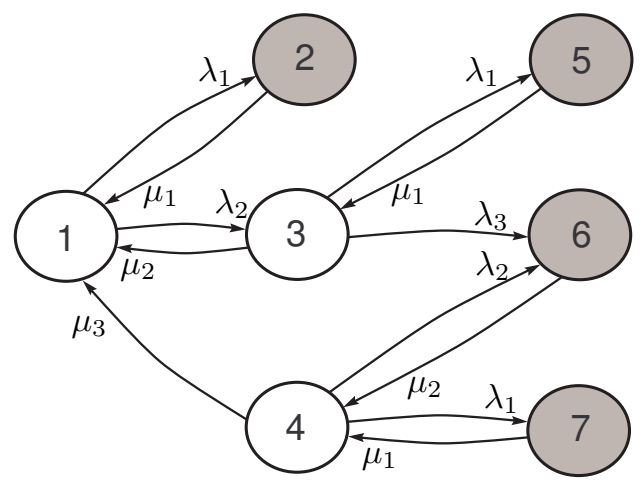

Figure 6. Markov diagram of system with priority repair of $\mathrm{C} 2$.
These changes in failure rates may conduct to a variation of the system availability. Our objective is to use the DIM in order to quantify the relative contribution of a component (a group of components), a state (or a group of states) of the system, on this variation.

\subsection{DIM for a component, a group of components}

A directional perturbation matrix $\mathbf{Q}_{\lambda_{i}}$ is first considered corresponding to changes in the direction of a single parameter of interest, e.g. the failure rate of component $i, \lambda_{i}$.

Uniform changes: Consider now the scenario H1, the results of DIMs in the directions $\mathbf{Q}_{\lambda_{i}}(i=1,2,3)$ are obtained by using the analytical expression (Equation 10). The results in Table 3 show that the contribution of $\mathrm{C} 1$ on the total variation of system availability is more important than that of $\mathrm{C} 2$ or $\mathrm{C} 3$, and the components'importance ranking is $\mathrm{C} 1>\mathrm{C} 2>\mathrm{C} 3$.

Table 3. DIM for components, case scenario H1.

\begin{tabular}{ccccc}
\hline Unit & $\operatorname{DIM}$ & Theoretic & Estimation & Ranking \\
\hline $\mathrm{C} 1$ & $\operatorname{DIM}\left(\mathbf{Q}_{\lambda_{1}}\right)$ & 0.6429 & 0.6380 & 1 \\
\hline $\mathrm{C} 2$ & $\operatorname{DIM}\left(\mathbf{Q}_{\lambda_{2}}\right)$ & 0.2143 & 0.2161 & 2 \\
\hline $\mathrm{C} 3$ & $\operatorname{DIM}\left(\mathbf{Q}_{\lambda_{3}}\right)$ & 0.1429 & 0.1459 & 3 \\
\hline
\end{tabular}

From the additivity property, the DIMs for the pairs of parameters are:

$\operatorname{DIM}\left(\mathbf{Q}_{\lambda_{1}}, \mathbf{Q}_{\lambda_{2}}\right)=\operatorname{DIM}\left(\mathbf{Q}_{\lambda_{1}}\right)+\operatorname{DIM}\left(\mathbf{Q}_{\lambda_{2}}\right)=0.8572$, $\operatorname{DIM}\left(\mathbf{Q}_{\lambda_{1}}, \mathbf{Q}_{\lambda_{3}}\right)=\operatorname{DIM}\left(\mathbf{Q}_{\lambda_{1}}\right)+\operatorname{DIM}\left(\mathbf{Q}_{\lambda_{3}}\right)=0.7858$, $\operatorname{DIM}\left(\mathbf{Q}_{\lambda_{2}}, \mathbf{Q}_{\lambda_{3}}\right)=\operatorname{DIM}\left(\mathbf{Q}_{\lambda_{2}}\right)+\operatorname{DIM}\left(\mathbf{Q}_{\lambda_{3}}\right)=0.3572$. According to these measures, the groups'/components' ranking is: $\mathrm{C} 3<\mathrm{C} 2<(\mathrm{C} 2, \mathrm{C} 3)<\mathrm{C} 1<(\mathrm{C} 1, \mathrm{C} 3)<(\mathrm{C} 1, \mathrm{C} 2)$.

Proportional relative changes: Table 4 represents the results of DIMs for the second scenario (H2). According to these importance measures, the components importance ranking could be drawn. More precisely, $\mathrm{C} 1$ becomes the less important one, and the most important component is $\mathrm{C} 2$.

Table 4. DIM for components, case scenario H2.

\begin{tabular}{ccccc}
\hline Unit & DIM & Theoretic & Estimation & Ranking \\
\hline $\mathrm{C} 1$ & $\operatorname{DIM}\left(\mathbf{Q}_{\lambda_{1}}\right)$ & 0.2647 & 0.2606 & 3 \\
\hline $\mathrm{C} 2$ & $\operatorname{DIM}\left(\mathbf{Q}_{\lambda_{2}}\right)$ & 0.4412 & 0.4414 & 1 \\
\hline $\mathrm{C} 3$ & $\operatorname{DIM}\left(\mathbf{Q}_{\lambda_{3}}\right)$ & 0.2941 & 0.2980 & 2 \\
\hline
\end{tabular}

Similarly, the DIMs for the pairs of parameters are: $\operatorname{DIM}\left(\mathbf{Q}_{\lambda_{1}}, \mathbf{Q}_{\lambda_{2}}\right)=\operatorname{DIM}\left(\mathbf{Q}_{\lambda_{1}}\right)+\operatorname{DIM}\left(\mathbf{Q}_{\lambda_{2}}\right)=0.7059$, $\operatorname{DIM}\left(\mathbf{Q}_{\lambda_{1}}, \mathbf{Q}_{\lambda_{3}}\right)=\operatorname{DIM}\left(\mathbf{Q}_{\lambda_{1}}\right)+\operatorname{DIM}\left(\mathbf{Q}_{\lambda_{3}}\right)=0.5588$, $\operatorname{DIM}\left(\mathbf{Q}_{\lambda_{2}}, \mathbf{Q}_{\lambda_{3}}\right)=\operatorname{DIM}\left(\mathbf{Q}_{\lambda_{2}}\right)+\operatorname{DIM}\left(\mathbf{Q}_{\lambda_{3}}\right)=0.7353$. Considering these results, the groups'/components' ranking is: $\mathrm{C} 1<\mathrm{C} 3<\mathrm{C} 2<(\mathrm{C} 1, \mathrm{C} 3)<(\mathrm{C} 1, \mathrm{C} 2)<(\mathrm{C} 2, \mathrm{C} 3)$. 
Note however that the components' ranking that we obtain are not absolute importance rankings, but the ranking relative to the DIM criterion with a specific scenario of changes. Obviously, different rankings could be obtained if a different importance measure was used.

\subsection{DIM for a given state}

To study the sensitivity of a given state, some specific directions of sensitivity are considered. In Tables 5 and $6, \mathbf{Q}_{i}$ represents the direction of all failure rate transitions leaving from the operational state $i(i=1,3,4)$. The $\operatorname{DIM}\left(\mathbf{Q}_{i}\right)$ provides the relative contribution of state $i$ on the total variation of system availability provoked by the changes in components' failure rates.

Table 5. DIM for states, case scenario H1.

\begin{tabular}{ccccc}
\hline State & DIM & Theoretic & Estimation & Ranking \\
\hline 1 & $\operatorname{DIM}\left(\mathbf{Q}_{1}\right)$ & 0.5714 & 0.5709 & 1 \\
\hline 3 & $\operatorname{DIM}\left(\mathbf{Q}_{3}\right)$ & 0.2857 & 0.2863 & 2 \\
\hline 4 & $\operatorname{DIM}\left(\mathbf{Q}_{4}\right)$ & 0.1429 & 0.1427 & 3 \\
\hline
\end{tabular}

The results of $\operatorname{DIM}\left(\mathbf{Q}_{i}\right)(i=1,3,4)$ for the case $\mathrm{H} 1$ (uniform changes) are reported in Table 5. According to $\operatorname{DIM}\left(\mathbf{Q}_{i}\right)$ 's measures, state 1 is the most important and state 3 is more important than state 4 . This importance ranking still holds for the case where the components' failure rates change proportionally (case H2), see Table 6 .

Table 6. DIM for states, case scenario H2.

\begin{tabular}{ccccc}
\hline State & DIM & Theoretic & Estimation & Ranking \\
\hline 1 & $\operatorname{DIM}\left(\mathbf{Q}_{1}\right)$ & 0.4706 & 0.4693 & 1 \\
\hline 3 & $\operatorname{DIM}\left(\mathbf{Q}_{3}\right)$ & 0.3529 & 0.3554 & 2 \\
\hline 4 & $\operatorname{DIM}\left(\mathbf{Q}_{4}\right)$ & 0.1765 & 0.1754 & 3 \\
\hline
\end{tabular}

\subsection{Estimation of DIM}

The exact results shown in previous paragraphs are obtained by using the analytical calculation under the hypothesis that the transition rate matrix $\mathbf{M}$ of Markov process is available. But in practical application, this assumption is not always true. Assume now that the $\mathbf{M}$ is unknown and that only available data are a single sample path of Markov process. Since the realistic data set of this trajectory is not available here, it need to be simulated with parameter values given in Table 1. The goal is to use the perturbation analysis technique to estimate the DIM's measures mentioned above from this data set. In fact, the simulation is made for 100000 transitions.

The steady state vector $\pi$ and the realization matrix D have been estimated by using Equations (16) and (18) respectively. All the results presented in Tables
3, 4,5 and 6 are obtained by changing only the directional matrix in Equation (20). The numerical results show that the estimated values are very close to those given by the analytical method.

To illustrate the convergence of the perturbation analysis approach, the Figure 7 sketches the evolution of the estimator $\operatorname{DIM}\left(\mathbf{Q}_{\lambda_{1}}\right)$ as a function of the sample size.

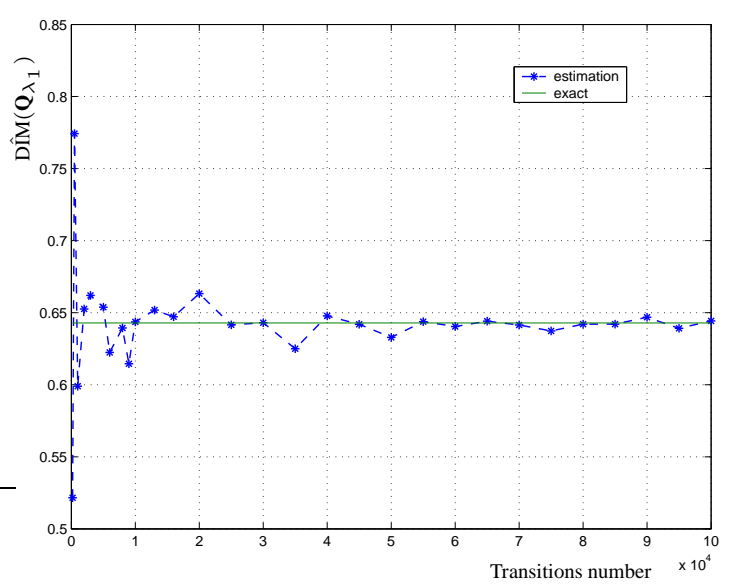

Figure 7. Estimator $\operatorname{DIM}\left(\mathbf{Q}_{\lambda_{1}}\right)$ as a function of the sample size

\section{CONCLUSIONS}

In this work, the differential importance measure (DIM) is extended to the dynamic systems including inter-components, functional dependencies, or more generally, systems described by Markov models. This allows to qualify the relative contribution of a component (or a group of components), as well as a given state (or a group of states) on the total variation of system performance provoked by the changes in system parameters' values.

In order to evaluate the DIM at steady state, both analytical method and estimation approach based on perturbation analysis are investigated. Numerical results show that the perturbation analysis technique can provide a very good estimation using only a single sample path of Markov process. From a practical point of view, this approach can be therefore a powerful tool to estimate DIM's measures from the operating feedback data of system without knowing components' reliability behavior (failure and/or repair rate,...), consequently, the transition rate matrix of Markov model.

Our further research focuses on the application of perturbation analysis to estimate the high-order extension of the DIM presented in (Do Van et al. 2008b). At last more detailed applications of these importance measures to decision-marking in reliability engineering, e.g. to the optimization of maintenance policies, are under study. 


\section{REFERENCES}

Borgonovo, E., G. Apostolakis, S. Tarantola, and A. Saltelli (2003). Comparison of global sensitivity analysis techniques and importance measures in PSA. Reliability Engineering and System Safety 79, 175-185.

Borgonovo, E. and G.-E. Apostolakis (2001). A new importance measure for risk-informed decision making. Reliability Engineering and System Safety 72(2), 193-212.

Cao, X.-R. and H.-F. Chen (1997). Pertubation realization, potentials, and sensitivity analysis of Markov processes. IEEE Transactions on Automatic Control 42(10), 1382-1393.

Cao, X.-R. and Y.-W. Wan (1998). Algorithms for sensitivity analysis of Markov systems through potential and perturbation realization. IEEE Transactions on Automatic Control 6(4), 482494.

Do Van, P., A. Barros, and C. Berenguer (2008a). Importance measure on finite time horizon and application to markovian multi-state production systems. Journal of Risk and Reliability 222, 449-461.

Do Van, P., A. Barros, and C. Berenguer (2008b). A new result on the differential importance measures of Markov systems. In Ninth International Probabilistic Safety Assessement and Management Conference - Proc.PSAM9, 18-23 may 2008, Hong Kong, China.

Do Van, P., A. Barros, and C. Berenguer (2008c). Reliability importance analysis of markovian systems at steady state using perturbation analysis. Reliability Engineering and System Safety 93(11), 1605-1615.

Marseguerra, M. and E. Zio (2004). Monte carlo estimation of the differential importance measure: application to the protection system of a nuclear reactor. Reliability Engineering and System Safety 86(1), 11-24.

Ou, Y. and J. Bechta-Dugan (2003). Approximate sensitivity analysis for acyclic Markov reliability models. IEEE Transactions on Reliability 52(2), 220-231.

Ross, S. (1996). Stochastic Processes. Wiley Series in Probability and Statistics. John Wiley \& Sons, Inc.

Vinod, G., H. Kushwaha, A. Verma, and A. Srividya (2003). Importance measures in ranking piping components for risk informed in-service inspection. Reliability Engineering and System Safety 80(2), 107-113.

Zio, E. and L. Podofillini (2006). Accounting for components interactions in the differential im- portance measure. Reliability Engineering and System Safety 91, 1163-1174. 